\title{
The association of CASC16 variants with breast Cancer risk in a northwest Chinese female population
}

\author{
Xiaoxiao Zuo ${ }^{*}$, Huanhuan Wang ${ }^{\dagger}$, Yin Mi, Yue Zhang, Xiaofei Wang, Ya Yang and Suna Zhai
}

\begin{abstract}
Purpose: Genetic variants play a critical role in the development of breast cancer. This investigation aimed to explore the association between CASC16 polymorphisms and breast cancer susceptibility.

Methods: We conducted a case-control study of 681 patients and 680 healthy individuals to investigate the correlation of five SNPs with breast cancer in a Northwest Chinese female population. Odds ratios (OR) and 95\% confidence intervals (Cls) were used to assess the association.

Results: Our study found that rs4784227 and rs 12922061 were significantly related to an increased susceptibility to breast cancer (OR 1.22, $p=0.022 ; \mathrm{OR} 1.21, p=0.026)$. While rs3803662 was a protective role in breast cancer risk (OR $0.69, p=0.042)$. Stratified analyses indicated that rs4784227 and rs 12922061 would increase breast cancer susceptibility at age > 50 years. Rs3803662 was a reduced factor of breast cancer risk by age $\leq 50$ years. Rs 4784227 was significantly increased risk of breast cancer in stage III/IV. The rs 45544231 and rs3112612 had a protective effect on breast cancer with tumor size $>2$ cm. Rs4784227 and rs12922061 could enhance breast cancer risk in lymph node metastasis positive individuals. CASC16 rs 12922061 and rs4784227 polymorphisms correlated with an increased risk of breast cancer in BMI > 24 $\mathrm{kg} / \mathrm{m}^{2}$. Haplotype analyses revealed that $\mathrm{G}_{\mathrm{rs} 45544231} T_{\mathrm{rs} 12922061} A_{\mathrm{rs} 3112612}$ and $\mathrm{G}_{\mathrm{rs} 45544231} \mathrm{C}_{\mathrm{rs} 12922061} A_{\mathrm{rs} 3112612}$ haplotypes decreased breast cancer risk.
\end{abstract}

Conclusion: Our study revealed that CASC16 genetic variants were significantly related to breast cancer susceptibility, which might give scientific evidence for exploring the molecular mechanism of breast cancer.

Keywords: Breast cancer, CSAC16, Polymorphism, Susceptibility

\section{Introduction}

Breast cancer $(\mathrm{BC})$ is one of the common malignant tumors in women (Torre et al. 2017) and the 2nd leading cause of cancer death among females in China (Chen et al. 2016a). The China National Cancer Centre recently reported that the incidence of breast cancer is $7.33 \%$ in China, of which $6.29 \%$ is in the northwest. Breast cancer showed a high mortality $(2.70 \%)$ and the highest incidence $(5.70 \%)$ rates in women of Northwest China. As of 2014, the newly increased incidence rates were $25.33,24.47$, and $11.28 \%$ among those aged $15-44$ years, $45-59$ years and 60-79 years, respectively (Wanqing et al. 2014; F B, et al.

\footnotetext{
* Correspondence: zuoxiao1985@163.com

${ }^{+}$Xiaoxiao Zuo and Huanhuan Wang contributed equally to this work. Department of Radiation Oncology, First Affiliated Hospital of Zhengzhou University, \#2 East Jianshe Road, Zhengzhou 450000, Henan, China
}

2018; Wan-qing et al. 2019). As a kind of multifactorial disease, $\mathrm{BC}$ is due to complex non-genetic and genetic factors (Rudolph 2016). Although non-genetic factors such as age, age of menarche, body mass index (BMI), procreative, and menstrual history were associated with an increased susceptibility to breast cancer (Anderson et al. 2004; Islam et al. 2013; Nelson et al. 2012; Zarco et al. 2012). Many recent studies have established that genetic factor also had a vital role in progression of breast cancer (Bray et al. 2013; Sehrawat et al. 2011; Ruiz-Narvaez et al. 2013; Han et al. 2011), and there were $27 \%$ of the breast cancer risk influenced by genetic variants (Lichtenstein et al. 2000). In addition, a number of genes including BRCA1, BRCA2, PTEN, TP53, CYP17 and other different genes have demonstrated that their polymorphisms were associated with risk of breast cancer (Nelson et al. 2012; 
Table 1 Characteristic of breast cancer patients and health control individuals

\begin{tabular}{llll}
\hline Variables & Cases $(n=681)$ & Controls $(n=680)$ & $p$ \\
\hline Age, years (mean \pm SD) & $50.58 \pm 9.84$ & $50.63 \pm 9.71$ & 0.930 \\
$\quad>50$ & $345(51 \%)$ & $344(51 \%)$ & \\
$\quad \leq 50$ & $336(49 \%)$ & $336(49 \%)$ & \\
Tumor position & & &
\end{tabular}

Tumor position

$\begin{array}{ll}\text { Left } & 274(40 \%) \\ \text { Right } & 288(42 \%) \\ \text { Missing } & 119(18 \%)\end{array}$

LN metastasis

Node-positive

$323(47 \%)$

Node-negative

$331(49 \%)$

Missing

$27(4 \%)$

Clinical stage

$\begin{array}{ll}\text { III/IV } & 150(22 \%) \\ \text { I/II } & 321(47 \%) \\ \text { Missing } & 210(31 \%) \\ \text { Tumor size } & \\ >2 \mathrm{~cm} & 409(60 \%) \\ \leq 2 \mathrm{~cm} & 139(20 \%) \\ \text { Missing } & 133(20 \%) \\ \text { PR } & \\ \text { Positive } & 414(61 \%)\end{array}$

Negative $257(38 \%)$

Missing $10(1 \%)$

ER

Positive $\quad 462$ (68\%)

Negative $198(29 \%)$

Missing $21(3 \%)$

C-erb

Positive $\quad 405(59 \%)$

Negative $\quad 114(17 \%)$

Missing $162(24 \%)$

Menopausal status

Yes $\quad 321(47 \%)$

No $\quad 247(36 \%)$

Missing $\quad 113(17 \%)$

Procreative times

1

227 (33\%)

$>1$

$260(38 \%)$

Missing

$194(29 \%)$

Age of menarche (years)

$\begin{array}{ll}\leq 14 & 340(50 \%) \\ >14 & 233(34 \%) \\ \text { Missing } & 108(16 \%)\end{array}$

Table 1 Characteristic of breast cancer patients and health control individuals (Continued)

\begin{tabular}{llll}
\hline Variables & Cases $(n=681)$ & Controls $(n=680)$ & $p$ \\
\hline $\mathrm{BMI}, \mathrm{kg} / \mathrm{m}^{2}{\text { (mean } \pm \mathrm{SD})^{\mathrm{a}}}^{\mathrm{a}}$ & & & \\
$\leq 24$ & $333(49 \%)$ & $240(35 \%)$ & 0.274 \\
$>24$ & $168(25 \%)$ & $114(17 \%)$ & 0.321 \\
Missing & $180(26 \%)$ & $326(48 \%)$ & \\
\hline
\end{tabular}

a Student's t-test is used. $p<0.05$ indicates statistical significance

$P R$ progesterone receptor, $E R$ estrogen receptor, $B M I$ body mass index, $L N$ lymph node

Liao et al. 2018; Walsh and King 2007; Han et al. 2016a; Wang et al. 2016; Lilyquist et al. 2018; He et al. 2014; Chen et al. 2016b; Yang et al. 2018).

Cancer-susceptibility candidate 16 gene (CASC16), also termed LOC643714, is a kind of long non-protein coding RNA and located at chromosome 16q12.1. Data from one study showed that CASC16 gene had a higher expression in breast cancer cells compared with normal cells (Han et al. 2016b). Furthermore, several studies had revealed a correlation between LOC643714 gene and BC (He et al. 2014; Ruiz-Narvaez et al. 2010; Low et al. 2013), but the functions of this gene are still unknown. Liao et al. found that rs12922061 polymorphism of the CASC16 gene was significantly increased susceptibility to breast cancer in southern China population (Liao et al. 2018). And the rs3803662 and rs12922061 also could increase the risk of breast cancer in a Japanese population (Low et al. 2013). However, another study indicated that rs4784227 of LOC643714 could improve BC risk, but rs3803662 and rs3112612 haven't observed a significant association in a southern Chinese population (He et al. 2014). The rs3803662 of LOC643714 also had no significant association with $\mathrm{BC}$ risk in AfricanAmerican women (Ruiz-Narvaez et al. 2010). These differences in the previous results may be due to the race, geographical location, lifestyle, and environmental exposure in specific Chinese population, which may be resulted in differences in the frequencies of genetic polymorphisms. As we all known, the Han Chinese population exhibits a complicated substructure, because the genes of northern China differ greatly from those of Southern China. However, the previous studies mainly focused on rs3803662, rs12922061, and rs3112612 polymorphisms in CASC16 association with breast cancer risk in a Southern Chinese population. The correlation between these three SNPs and breast cancer hadn't been identified in the Northwest Chinese population.

In this case-control study, we selected five SNPs (rs3803662, rs4784227, rs45544231, rs12922061, and rs3112612) in the CASC16 gene according to the previous studies and the 1000 genomes project. We further investigated the association between CASC16 genetic variants and $\mathrm{BC}$ susceptibility in a Northwest Chinese female population. 
Table 2 The distribution of allele frequencies of CASC16 SNPs in case and control

\begin{tabular}{|c|c|c|c|c|c|c|c|c|c|}
\hline \multirow[t]{2}{*}{ SNP ID } & \multirow{2}{*}{$\begin{array}{l}\text { Alleles } \\
\text { (minor/ } \\
\text { major) }\end{array}$} & \multirow{2}{*}{$\begin{array}{l}\text { Chromosome } \\
\text { position }\end{array}$} & \multicolumn{2}{|l|}{ MAF } & \multirow{2}{*}{$\begin{array}{l}\mathrm{O} \\
(\mathrm{HET})\end{array}$} & \multirow{2}{*}{$\begin{array}{l}\text { E } \\
(\text { HET) }\end{array}$} & \multirow{2}{*}{$\begin{array}{l}p^{a}- \\
H W E\end{array}$} & \multirow[t]{2}{*}{ OR $(95 \% \mathrm{Cl})$} & \multirow[t]{2}{*}{$p^{b}$} \\
\hline & & & Case & Control & & & & & \\
\hline rs3803662 & $\mathrm{G} / \mathrm{A}$ & chr16: 52552429 & 0.307 & 0.328 & 0.430 & 0.441 & 0.542 & $0.91(0.77-1.06)$ & 0.228 \\
\hline rs4784227 & $\mathrm{T} / \mathrm{C}$ & chr16: 52565276 & 0.277 & 0.239 & 0.356 & 0.363 & 0.596 & $1.22(1.03-1.45)$ & 0.022 \\
\hline rs45544231 & $\mathrm{C} / \mathrm{G}$ & chr16: 52598818 & 0.197 & 0.193 & 0.302 & 0.312 & 0.389 & $1.02(0.85-1.24)$ & 0.824 \\
\hline rs12922061 & $\mathrm{T} / \mathrm{C}$ & chr16: 52601088 & 0.285 & 0.247 & 0.385 & 0.372 & 0.410 & $1.21(1.02-1.44)$ & 0.026 \\
\hline rs3112612 & $\mathrm{G} / \mathrm{A}$ & chr16: 52601252 & 0.197 & 0.195 & 0.299 & 0.314 & 0.221 & $1.01(0.84-1.23)$ & 0.885 \\
\hline
\end{tabular}

SNP single nucleotide polymorphisms, MAF minor allele frequency, HWE Hardy-Weinberg equilibrium

$p^{\mathrm{a}}$ values were calculated by exact test, $p^{\mathrm{a}}<0.05$ are excluded

$p^{b}$ values were calculated by two-sided $x^{2}, p^{b}<0.05$ indicates statistical significance

Our findings would give available information for prevention and management of breast cancer.

\section{Materials and methods}

\section{Study population}

In this present case-control study, 681 unrelated Chinese female breast cancer patients and 680 healthy subjects were recruited from the Shaanxi Provincial Cancer Hospital. All patients were newly diagnosed with histological examination and confirmed to be BC. Patients with a history of autoimmunity, secondary tumors, severe infections diseases, other types of cancer and family history of any cancers included breast cancer were excluded. Healthy individuals were matched with the case subjects based on age and ethnicity, who were randomly selected from the cancer-free female population with a routine health examination in the same hospital. Controls with the family history of any cancers were excluded. Each study participant was informed the purpose of the sample collection and their written consent were obtained. The participants' basic information were obtained from the patients or their medical records including age, ethnicity, place of residence, tutor position, lymph node metastasis status, clinical stage, tumor size, estrogenic receptor (ER), progesterone receptor (PR) status, menopausal status, procreative times, age of menarche, and body mass index (BMI). All experiments were carried out depending on the guideline of Helsinki's declaration and our study were approved by the ethics committee of the Shaanxi Provincial Cancer Hospital.

\section{Selection of SNPs and genotype analysis}

We selected five polymorphisms of CASC16 in the present study. Of the five SNPs, three polymorphisms (rs3803662, rs12922061, and rs3112612) were chosen basing upon the published papers which they reported that these SNPs might be related to breast cancer susceptibility (He et al. 2014). While rs4784227 and rs45544231 were obtained from the 1000 Genomes Project with a minor allele frequency (MAF) $>5 \%$ for further genotype. We extracted genomic DNA from peripheral blood samples from the study participants using a blood genomic DNA extraction kit (GoldMag,
Xi'an, China). NanoDrop 2000C spectrophotometer (Thermo Scientific, Waltham, USA) were implemented to check purity and concentration of the genomic DNA and then kept at $-20^{\circ} \mathrm{C}$ for further analysis. We used Agena Bioscience Assay Design Suite V2.0 software (https://agenacx. com/online-tools/) to design PCR primers. SNP genotype was identified by Agena MassARRAY iPLEX platform, and Agena Bioscience TYPER version 4.0 software was used to manage and analyze the data (Xia et al. 2014; Zhou et al. 2015). To validate the genotype results, $10 \%$ of samples were randomly selected, and genotypes showed $100 \%$ concordance for all SNPs according to Sanger sequencing.

\section{Statistical analysis}

The differences in demographic characteristics between the case and control group were analyzed by continuous variable independent sample t-test and category variable Pearson's chi-square test. Hardy-Weinberg equilibrium (HWE) of each SNP was tested by chi-squared test to assess genotype frequencies in controls. Comparisons of distribution in SNP allele and genotype frequencies between case and control were checked by a Pearson chi-squared test or Fisher's exact test. The association between CASC16 SNPs and BC susceptibility were assessed by computing odds ratios (ORs) and 95\% confidence intervals (CIs) in five inheritance models (allele, co-dominant, dominant, recessive, and log-additive) using logistic regression analysis with or without adjustment for age or BMI. Linkage disequilibrium (LD) was constructed by Haploview V4.2 software and haplotype was analyzed by logistic regression. Besides, we also evaluated the relationship between CASC16 polymorphisms and $\mathrm{BC}$ patient subgroups with stratification analyses. All statistical analyses were performed using SPSS version 17.0 software (IBM Analytics, Chicago, IL) and PLINK software. All statistical tests were two-tailed and $p$-value $<0.05$ was considered statistical significance.

\section{Results}

Characteristics of the study population

The basic information of the study subjects was summarized in Table 1 . The average ages were $50.58 \pm 9.84$ years 
Table 3 Association between CASC16 polymorphisms and breast cancer risk

\begin{tabular}{|c|c|c|c|c|c|c|}
\hline SNP ID & Model & Genotype & $\begin{array}{l}\text { Case } \\
\mathrm{N}(\%)\end{array}$ & $\begin{array}{l}\text { Control } \\
\mathrm{N}(\%)\end{array}$ & OR (95\% Cl) & $p^{a}$ \\
\hline \multicolumn{7}{|c|}{ rs3803662 } \\
\hline & Codominant & A/A & $318(46.70)$ & $310(45.66)$ & 1 & \\
\hline & & $A / G$ & $308(45.23)$ & $292(43.00)$ & $1.03(0.82-1.29)$ & 0.805 \\
\hline & & $\mathrm{G} / \mathrm{G}$ & $55(8.08)$ & $77(11.34)$ & $0.70(0.48-1.02)$ & 0.061 \\
\hline & Dominant & A/A & $318(46.70)$ & $310(45.66)$ & 1 & \\
\hline & & $A / G-G / G$ & 363 (53.30) & 369 (54.34) & $0.96(0.77-1.18)$ & 0.700 \\
\hline & Recessive & A/A-A/G & 626 (91.92) & $602(88.66)$ & 1 & \\
\hline & & $\mathrm{G} / \mathrm{G}$ & $55(8.08)$ & $77(11.34)$ & $0.69(0.48-0.99)$ & 0.042 \\
\hline & Log-additive & - & - & - & $0.90(0.77-1.06)$ & 0.223 \\
\hline \multicolumn{7}{|c|}{ rs4784227 } \\
\hline & Codominant & $\mathrm{C} / \mathrm{C}$ & $353(52.30)$ & $394(58.37)$ & 1 & \\
\hline & & $\mathrm{T} / \mathrm{C}$ & $270(40.00)$ & $240(35.56)$ & $1.26(1.00-1.57)$ & 0.048 \\
\hline & & $T / T$ & $52(7.70)$ & $41(6.07)$ & $1.42(0.92-2.18)$ & 0.117 \\
\hline & Dominant & $C / C$ & $353(52.30)$ & $394(58.37)$ & 1 & \\
\hline & & $\mathrm{T} / \mathrm{C}-\mathrm{T} / \mathrm{T}$ & $322(47.70)$ & $281(41.63)$ & $1.28(1.03-1.59)$ & 0.025 \\
\hline & Recessive & C/C-T/C & $623(92.30)$ & $634(93.93)$ & 1 & \\
\hline & & $\mathrm{T} / \mathrm{T}$ & $52(7.70)$ & $41(6.07)$ & $1.29(0.84-1.97)$ & 0.239 \\
\hline & Log-additive & - & - & - & $1.22(1.03-1.45)$ & 0.023 \\
\hline \multicolumn{7}{|c|}{ rs45544231 } \\
\hline & Codominant & $\mathrm{G} / \mathrm{G}$ & 445 (65.35) & $446(65.59)$ & 1 & \\
\hline & & $\mathrm{G} / \mathrm{C}$ & 204 (29.96) & $205(30.15)$ & $0.99(0.79-1.26)$ & 0.980 \\
\hline & & $\mathrm{C} / \mathrm{C}$ & $32(4.70)$ & $29(4.26)$ & $1.11(0.66-1.86)$ & 0.707 \\
\hline & Dominant & $\mathrm{G} / \mathrm{G}$ & 445 (65.35) & 446 (65.59) & 1 & \\
\hline & & $\mathrm{G} / \mathrm{C}-\mathrm{C} / \mathrm{C}$ & $236(34.65)$ & $234(34.41)$ & $1.01(0.81-1.26)$ & 0.928 \\
\hline & Recessive & $\mathrm{G} / \mathrm{G}-\mathrm{G} / \mathrm{C}$ & 649 (95.30) & $651(95.74)$ & 1 & \\
\hline & & $C / C$ & $32(4.70)$ & $29(4.26)$ & $1.11(0.66-1.85)$ & 0.701 \\
\hline & Log-additive & - & - & - & $1.02(0.85-1.23)$ & 0.831 \\
\hline \multicolumn{7}{|c|}{ rs12922061 } \\
\hline & Codominant & $\mathrm{C} / \mathrm{C}$ & $348(51.10)$ & $381(56.03)$ & 1 & \\
\hline & & $C / T$ & $278(40.82)$ & $262(38.53)$ & $1.16(0.93-1.45)$ & 0.187 \\
\hline & & $T / T$ & $55(8.08)$ & 37 (5.44) & $1.63(1.05-2.53)$ & 0.030 \\
\hline & Dominant & $\mathrm{C} / \mathrm{C}$ & $348(51.10)$ & $381(56.03)$ & 1 & \\
\hline & & $\mathrm{C} / \mathrm{T}-\mathrm{T} / \mathrm{T}$ & $333(48.90)$ & $299(43.97)$ & $1.22(0.99-1.51)$ & 0.068 \\
\hline & Recessive & $\mathrm{C} / \mathrm{C}-\mathrm{C} / \mathrm{T}$ & 626 (91.92) & $643(94.56)$ & 1 & \\
\hline & & $\mathrm{T} / \mathrm{T}$ & $55(8.08)$ & $37(5.44)$ & $1.53(0.99-2.35)$ & 0.054 \\
\hline & Log-additive & - & - & - & $1.22(1.03-1.45)$ & 0.025 \\
\hline \multicolumn{7}{|c|}{ rs3112612 } \\
\hline & Codominant & A/A & 444 (65.29) & $446(65.59)$ & 1 & \\
\hline & & $A / G$ & $204(30.00)$ & $203(29.85)$ & $1.01(0.80-1.28)$ & 0.938 \\
\hline & & $\mathrm{G} / \mathrm{G}$ & $32(4.71)$ & $31(4.56)$ & $1.04(0.62-1.73)$ & 0.891 \\
\hline & Dominant & A/A & 444 (65.29) & $446(65.59)$ & 1 & \\
\hline & & $A / G-G / G$ & $236(34.71)$ & $234(34.41)$ & $1.01(0.81-1.27)$ & 0.911 \\
\hline & Recessive & $A / A-A / G$ & 648 (95.29) & 649 (95.44) & 1 & \\
\hline & & $\mathrm{G} / \mathrm{G}$ & $32(4.71)$ & $31(4.56)$ & $1.03(0.62-1.72)$ & 0.899 \\
\hline & Log-additive & - & - & - & $1.01(0.84-1.22)$ & 0.889 \\
\hline
\end{tabular}

Cl confidence interval, OR odds ratio, SNP single nucleotide polymorphism 
in cases and $50.63 \pm 9.71$ years in controls. There was no significant difference in age between the case and control group $(p=0.930)$.

\section{Association between CASC16 polymorphisms and BC risk} Five SNPs in the CASC16 gene were selected and analysed in this case-control study. The distribution of allele frequencies between cases and controls was compared using chi-square test (Table 2). All five SNPs conformed to the HWE among controls $(p>0.05)$. It means appropriate SNP selection. And our results showed that the minor allele of two SNPs (rs4784227 and rs12922061) were significantly associated with increased $\mathrm{BC}$ susceptibility under allele model $(\mathrm{OR}=1.22,95 \% \mathrm{CI}=1.03-1.45, p=$ 0.022 ; $\mathrm{OR}=1.21,95 \% \mathrm{CI}=1.02-1.44, p=0.026$, respectively). We further examined the correlation between the genotypes of SNPs and BC risk by logistic regression analysis with adjustments for age under the codominant, dominant, recessive, and log-additive models (Table 3). We found that rs4784227 was related to a higher risk of $\mathrm{BC}$ in codominant model ( $\mathrm{T} / \mathrm{C}$ genotype, $\mathrm{OR}=1.26,95 \%$ $\mathrm{CI}=1.00-1.57, p=0.048)$, dominant model $(\mathrm{T} / \mathrm{C}-\mathrm{T} / \mathrm{T}$ genotype, $\mathrm{OR}=1.28,95 \% \mathrm{CI}=1.03-1.59, p=0.025)$ and the log-additive model $(\mathrm{OR}=1.22,95 \% \mathrm{CI}=1.03-1.45$, $p=0.023)$. The rs12922061 also had a significant higher susceptibility to $\mathrm{BC}$ in codominant model ( $\mathrm{T} / \mathrm{T}$ genotype, $\mathrm{OR}=1.63,95 \% \mathrm{CI}=1.05-2.53, p=0.030)$ and log-additive model $(\mathrm{OR}=1.22,95 \% \mathrm{CI}=1.03-1.45, p=0.025)$. In contrast, rs3803662 was associated with a reduced risk of $\mathrm{BC}$ in recessive model $(\mathrm{G} / \mathrm{G}$ genotype, $\mathrm{OR}=0.69,95 \% \mathrm{CI}=$ 0.48-0.99, $p=0.042$ ). Two SNPs (rs45544231 and rs3112612) were not observed association under any of the genetic models.

\section{Stratified analyses between SNPs and BC risk based on age and clinical characteristics}

The association between five SNPs and BC susceptibility was analyzed by logistic regression under age and clinical characteristic subgroups (Tables 4 and 5). On age-based stratification, rs4784227 would significantly increase risk of $\mathrm{BC}$ in allele model $(\mathrm{OR}=1.34,95 \% \mathrm{CI}=1.10-1.79, p=0.007)$, codominant model $(\mathrm{T} / \mathrm{C}$ genotype, $\mathrm{OR}=1.46,95 \% \mathrm{CI}=$ 1.06-1.99, $p=0.019$ ), dominant model (T/C-T/T genotype, $\mathrm{OR}=1.51,95 \% \mathrm{CI}=1.11-2.04, p=0.008)$ and log-additive model $(\mathrm{OR}=1.42,95 \% \mathrm{CI}=1.10-1.82, p=0.006)$ of the patients at age $>50$ years (Table 4). And rs12922061 was also associated with an increased susceptibility to $\mathrm{BC}$ in allele model $(\mathrm{OR}=1.36,95 \% \mathrm{CI}=1.07-1.73, p=0.012)$, codominant model ( $\mathrm{T} / \mathrm{T}$ genotype, $\mathrm{OR}=1.91,95 \% \mathrm{CI}=1.04-3.51$, $p=0.036)$, dominant model $(\mathrm{C} / \mathrm{T}-\mathrm{T} / \mathrm{T}$ genotype, $\mathrm{OR}=1.41$, $95 \% \mathrm{CI}=1.05-1.91, p=0.024)$, and log-additive model (OR = $1.36,95 \% \mathrm{CI}=1.07-1.73, p=0.012$ ) in subjects $>50$ years old. However, the G/G genotype of rs3803662 played a reduced role in risk of breast cancer under the recessive model
$(\mathrm{OR}=0.53,95 \% \mathrm{CI}=0.32-0.88, p=0.014)$ of the patients $\leq 50$ years. We also assessed the effect of CASC16 gene polymorphisms on $\mathrm{BC}$ risk by clinical characteristics including clinical stage, tumor size, lymph node metastasis, and BMI. As was displayed in Table 5, it was found that T/T genotype of rs4784227 significantly improved risk of stage III/IV breast cancer patients $(\mathrm{OR}=2.19,95 \% \mathrm{CI}=1.08-4.46, p=0.031)$ compared with stage I/II. The allele ' $\mathrm{C}$ ' and $\mathrm{C} / \mathrm{C}$ genotype of rs45544231, allele ' $G$ ' and $G / G$ genotype of rs3112612 had protective effect on susceptibility of breast cancer with tumor size $>2 \mathrm{~cm}(\mathrm{OR}=0.72, p=0.045 ; \mathrm{OR}=0.29, p=0.001$; $\mathrm{OR}=$ $0.71, p=0.039 ; \mathrm{OR}=0.28, p=0.001$; respectively) than of tumor size $\leq 2 \mathrm{~cm}$. The results further confirmed that TC+ TT genotype of rs4784227 was significantly associated with an increased BC risk in lymph node metastasis positive individuals $(\mathrm{OR}=1.41,95 \% \mathrm{CI}=1.04-1.93, p=0.028)$. Minor allele ' $\mathrm{T}$ ' of rs12922061 was also noted to improve BC susceptibility in lymph node metastasis positive participants $(\mathrm{OR}=1.30,95 \% \mathrm{CI}=1.02-1.65, p=0.034)$. In addition, the CASC16 polymorphisms correlations with breast cancer were carried out in accordance with BMI-based stratification (Table 6). The results indicated that CASC16 rs12922061 and rs4784227 polymorphisms were significantly correlated with increased risk of breast cancer in BMI $>24 \mathrm{~kg} / \mathrm{m}^{2}$ subjects $(\mathrm{T}, \mathrm{OR}=1.54,95 \% \mathrm{CI}=1.05-2.26, p=0.026$; TT genotype, $\mathrm{OR}=13.41,95 \% \mathrm{CI}=1.74-103.6, p=0.013 ; \mathrm{T}$, $\mathrm{OR}=1.49,95 \% \mathrm{CI}=1.01-2.20, p=0.042$; respectively).

\section{Haplotype analyses of CASC16 polymorphisms and breast cancer risk}

We further examined the linkage disequilibrium (LD) and haplotype analyses of CASC16 polymorphisms in case and control subjects via Haploview software and logistic regression. The LD plot was shown in Fig. 1, and LD block was consisted of three SNPs including rs45544231, rs12922061 and rs3112612. The haplotype analysis revealed that $G_{r s 45544231} \mathrm{~T}_{\mathrm{rs} 12922061} \mathrm{~A}_{\mathrm{rs} 3112612}$ and $\mathrm{G}_{\mathrm{rs} 45544231} \mathrm{C}_{\mathrm{rs} 12922061} \mathrm{~A}_{\mathrm{rs} 3112612}$ haplotypes in the CASC16 gene were found to reduce risk of breast cancer $(\mathrm{OR}=0.82,95 \% \mathrm{CI}=0.69-0.98, p=0.025 ; \quad \mathrm{OR}=0.85$, 95\% CI $=0.73-0.99, p=0.039$; respectively; Table 7).

\section{Discussion}

In the present case-control study, 681 breast cancer patients and 680 free-cancer subjects were recruited to evaluate the correlation between CASC16 variants and $\mathrm{BC}$ risk in a Northwest Chinese female population. The research showed that CASC16 polymorphisms (rs4784227, rs12922061, and rs3803662) were significantly associated with BC susceptibility. Furthermore, rs4784227, rs12922061, rs3803662, rs45544231, and rs3112612 polymorphisms were associated with breast cancer patients with stratified subgroups including age, lymph node metastasis status, clinical 
Table 4 The relationship of CASC16 polymorphisms with breast cancer according to the stratification analysis by age

\begin{tabular}{|c|c|c|c|c|c|c|c|c|c|c|}
\hline \multirow[t]{2}{*}{ SNP } & \multirow[t]{2}{*}{ Model } & \multirow[t]{2}{*}{ Genotype } & \multicolumn{4}{|l|}{$>50$ years } & \multicolumn{4}{|l|}{$\leq 50$ years } \\
\hline & & & Case & Control & OR $(95 \% \mathrm{Cl})$ & $p$ & Case & Control & OR $(95 \% \mathrm{Cl})$ & $p$ \\
\hline \multirow[t]{10}{*}{ rs3803662 } & Allele & A & 486 (70.43\%) & 464 (67.64\%) & 1 & & $458(68.15 \%)$ & 448 (66.67\%) & 1 & \\
\hline & & G & 204 (29.57\%) & 222 (32.36\%) & $0.88(0.70-1.10)$ & 0.262 & 214(31.85\%) & 224(33.33\%) & $0.93(0.74-1.17)$ & 0.561 \\
\hline & Codominant & $\mathrm{A} / \mathrm{A}$ & 169 (48.99\%) & 151 (44.02\%) & 1 & & 149 (44.34\%) & 159 (47.32\%) & 1 & \\
\hline & & $A / G$ & 148 (42.90\%) & 162 (47.23\%) & $0.82(0.60-1.12)$ & 0.202 & $160(47.62 \%)$ & 130 (38.69\%) & $1.31(0.95-1.81)$ & 0.097 \\
\hline & & $\mathrm{G} / \mathrm{G}$ & $28(8.12 \%)$ & $30(8.75 \%)$ & $0.83(0.48-1.46)$ & 0.526 & 27 (8.04\%) & 47 (13.99\%) & $0.61(0.36-1.03)$ & 0.063 \\
\hline & Dominant & $\mathrm{A} / \mathrm{A}$ & 169 (48.99\%) & 151 (44.02\%) & 1 & & 149 (44.34\%) & 159 (47.32\%) & 1 & \\
\hline & & A/G-G/G & 176 (51.01\%) & 192 (55.98\%) & $0.82(0.61-1.11)$ & 0.191 & $187(55.65 \%)$ & 177 (52.68\%) & $1.13(0.83-1.53)$ & 0.443 \\
\hline & Recessive & $\mathrm{A} / \mathrm{A}-\mathrm{A} / \mathrm{G}$ & 317 (91.88\%) & 313 (91.25\%) & 1 & & 309 (91.96\%) & 289 (86.01\%) & 1 & \\
\hline & & $\mathrm{G} / \mathrm{G}$ & $28(8.12 \%)$ & $30(8.75 \%)$ & $0.92(0.54-1.58)$ & 0.768 & 27 (8.04\%) & 47 (13.99\%) & $0.53(0.32-0.88)$ & 0.014 \\
\hline & Log-additive & - & - & - & $0.87(0.69-1.10)$ & 0.249 & - & - & $0.93(0.74-1.17)$ & 0.553 \\
\hline \multirow[t]{10}{*}{ rs4784227 } & Allele & C & 489 (71.28\%) & 531 (77.63\%) & 1 & & 487 (73.34\%) & 497 (74.62\%) & 1 & \\
\hline & & $\mathrm{T}$ & 197 (28.72\%) & 153 (22.37\%) & $1.34(1.10-1.79)$ & 0.007 & 177 (26.66\%) & 169 (25.38\%) & $1.07(0.84-1.37)$ & 0.594 \\
\hline & Codominant & $\mathrm{C} / \mathrm{C}$ & 171 (49.85\%) & 205 (59.94\%) & 1 & & $182(54.82 \%)$ & 189 (56.76\%) & 1 & \\
\hline & & $\mathrm{T} / \mathrm{C}$ & 147 (42.86\%) & $121(35.38 \%)$ & $1.46(1.06-1.99)$ & 0.019 & $123(37.05 \%)$ & 119 (35.74\%) & $1.07(0.78-1.49)$ & 0.665 \\
\hline & & $\mathrm{T} / \mathrm{T}$ & 25 (7.29\%) & $16(4.68 \%)$ & $1.88(0.97-3.64)$ & 0.061 & 27 (8.13\%) & $25(7.51 \%)$ & $1.13(0.63-2.03)$ & 0.678 \\
\hline & Dominant & $\mathrm{C} / \mathrm{C}$ & 171 (49.85\%) & 205 (59.94\%) & 1 & & $182(54.82 \%)$ & 189 (56.76\%) & 1 & \\
\hline & & $\mathrm{T} / \mathrm{C}-\mathrm{T} / \mathrm{T}$ & $172(50.14)$ & 137 (40.06\%) & $1.51(1.11-2.04)$ & 0.008 & $150(45.18 \%)$ & 144 (43.24\%) & $1.08(0.80-1.47)$ & 0.606 \\
\hline & Recessive & C/C-T/C & 318 (92.71\%) & 326 (95.32\%) & 1 & & 305 (91.87\%) & 308 (92.49\%) & 1 & \\
\hline & & $\mathrm{T} / \mathrm{T}$ & $25(7.29 \%)$ & $16(4.68 \%)$ & $1.61(0.84-3.07)$ & 0.151 & 27 (8.13\%) & $25(7.51 \%)$ & $1.10(0.62-1.94)$ & 0.743 \\
\hline & Log-additive & - & - & - & $1.42(1.10-1.82)$ & 0.006 & - & - & $1.07(0.84-1.36)$ & 0.589 \\
\hline \multirow[t]{10}{*}{ rs45544231 } & Allele & G & 569 (82.46\%) & 563 (81.83\%) & 1 & & 525 (78.13\%) & $534(79.46 \%)$ & 1 & \\
\hline & & C & 121 (17.54\%) & 125 (18.17\%) & $0.96(0.73-1.26)$ & 0.759 & 147 (21.88\%) & 138 (20.54\%) & $1.08(0.83-1.41)$ & 0.548 \\
\hline & Codominant & $\mathrm{G} / \mathrm{G}$ & 239 (69.27\%) & 230 (66.86\%) & 1 & & 206 (61.31\%) & 216 (64.29\%) & 1 & \\
\hline & & $\mathrm{G} / \mathrm{C}$ & 91 (26.38\%) & 103 (29.94\%) & $0.85(0.61-1.19)$ & 0.341 & $113(33.63 \%)$ & 102 (30.36\%) & $1.16(0.83-1.61)$ & 0.377 \\
\hline & & $\mathrm{C} / \mathrm{C}$ & 15 (4.35\%) & $11(3.20 \%)$ & $1.31(0.59-2.92)$ & 0.504 & $17(5.06 \%)$ & $18(5.36 \%)$ & $0.98(0.49-1.96)$ & 0.964 \\
\hline & Dominant & $\mathrm{G} / \mathrm{G}$ & 239 (69.27\%) & 230 (66.86\%) & 1 & & 206 (61.31\%) & 216 (64.29\%) & 1 & \\
\hline & & $\mathrm{G} / \mathrm{C}-\mathrm{C} / \mathrm{C}$ & 106 (30.72\%) & 114 (33.14\%) & $0.89(0.65-1.23)$ & 0.496 & $13(38.69 \%)$ & 120 (35.71\%) & $1.13(0.83-1.55)$ & 0.433 \\
\hline & Recessive & $\mathrm{G} / \mathrm{G}-\mathrm{G} / \mathrm{C}$ & 330 (95.65\%) & 333 (96.80\%) & 1 & & 31 (94.94\%) & 318 (94.64\%) & 1 & \\
\hline & & $\mathrm{C} / \mathrm{C}$ & 15 (4.35\%) & $11(3.20 \%)$ & $1.34(0.62-3.04)$ & 0.429 & 17 (5.06\%) & $18(5.36 \%)$ & $0.94(0.47-1.85)$ & 0.849 \\
\hline & Log-additive & - & - & - & $0.96(0.73-1.26)$ & 0.763 & - & - & $1.08(0.83-1.39)$ & 0.568 \\
\hline \multirow[t]{10}{*}{ rs12922061 } & Allele & C & 485 (70.29\%) & 525 (76.31\%) & 1 & & 48 (72.77\%) & 499 (74.26\%) & 1 & \\
\hline & & $\mathrm{T}$ & 205 (29.71\%) & 163 (23.69\%) & $1.36(1.07-1.73)$ & 0.012 & $18(27.23 \%)$ & 173 (25.74\%) & $1.08(0.85-1.38)$ & 0.537 \\
\hline & Codominant & $\mathrm{C} / \mathrm{C}$ & $171(49.57 \%)$ & 200 (58.14\%) & 1 & & $17(52.68 \%)$ & 181 (53.87\%) & 1 & \\
\hline & & $\mathrm{C} / \mathrm{T}$ & $143(41.45 \%)$ & 125 (36.34\%) & $1.34(0.98-1.83)$ & 0.070 & 135(40.18\%) & 137 (40.77\%) & $1.01(0.74-1.38)$ & 0.953 \\
\hline & & $\mathrm{T} / \mathrm{T}$ & 31 (8.99\%) & $19(5.52 \%)$ & $1.91(1.04-3.51)$ & 0.036 & $24(7.14 \%)$ & $18(5.36 \%)$ & $1.37(0.72-2.61)$ & 0.340 \\
\hline & Dominant & $\mathrm{C} / \mathrm{C}$ & 171 (49.57\%) & 200 (58.14\%) & 1 & & $177(52.68 \%)$ & 181 (53.87\%) & 1 & \\
\hline & & $\mathrm{C} / \mathrm{T}-\mathrm{T} / \mathrm{T}$ & $174(50.43 \%)$ & 144 (41.86\%) & $1.41(1.05-1.91)$ & 0.024 & $159(47.32 \%)$ & 155 (46.13\%) & $1.05(0.78-1.42)$ & 0.747 \\
\hline & Recessive & $\mathrm{C} / \mathrm{C}-\mathrm{C} / \mathrm{T}$ & 314 (91.01\%) & 325 (94.48\%) & 1 & & 312 (92.86\%) & 318 (94.64\%) & 1 & \\
\hline & & $\mathrm{T} / \mathrm{T}$ & 31 (8.99\%) & $19(5.52 \%)$ & $1.69(0.94-3.06)$ & 0.082 & $24(7.14 \%)$ & $18(5.36 \%)$ & $1.36(0.73-2.56)$ & 0.335 \\
\hline & Log-additive & - & - & - & $1.36(1.07-1.73)$ & 0.012 & - & - & $1.09(0.85-1.39)$ & 0.518 \\
\hline \multirow[t]{3}{*}{ rs3112612 } & Allele & A & 569 (82.46\%) & $562(81.69 \%)$ & 1 & & $523(78.06 \%)$ & $533(79.32 \%)$ & 1 & \\
\hline & & G & $121(17.54 \%)$ & $126(18.31 \%)$ & $0.95(0.72-1.25)$ & 0.707 & 147 (21.94\%) & 139 (20.68\%) & $1.08(0.83-1.40)$ & 0.574 \\
\hline & Codominant & $\mathrm{A} / \mathrm{A}$ & 239 (69.28\%) & 230 (66.86\%) & 1 & & 205 (61.19\%) & 216 (64.29\%) & 1 & \\
\hline
\end{tabular}


Table 4 The relationship of CASC16 polymorphisms with breast cancer according to the stratification analysis by age (Continued)

\begin{tabular}{|c|c|c|c|c|c|c|c|c|c|c|}
\hline \multirow[t]{2}{*}{ SNP } & \multirow[t]{2}{*}{ Model } & \multirow[t]{2}{*}{ Genotype } & \multicolumn{4}{|l|}{$>50$ years } & \multicolumn{4}{|l|}{$\leq 50$ years } \\
\hline & & & Case & Control & OR $(95 \% \mathrm{Cl})$ & $p$ & Case & Control & OR $(95 \% \mathrm{Cl})$ & $p$ \\
\hline & & $A / G$ & 91 (26.38\%) & 102 (29.65\%) & $0.86(0.61-1.20)$ & 0.372 & $113(33.73 \%)$ & 101 (30.06\%) & $1.18(0.85-1.64)$ & 0.331 \\
\hline & & $\mathrm{G} / \mathrm{G}$ & 15 (4.35\%) & 12 (3.49\%) & $1.20(0.55-2.63)$ & 0.641 & 17 (5.07\%) & 19 (5.65\%) & $0.94(0.47-1.85)$ & 0.848 \\
\hline & Dominant & $\mathrm{A} / \mathrm{A}$ & 239 (69.28\%) & 230 (66.86\%) & 1 & & 205 (61.19\%) & $216(64.29 \%)$ & 1 & \\
\hline & & A/G-G/G & 106 (30.72\%) & 114 (33.14\%) & $0.89(0.65-1.23)$ & 0.496 & $130(38.81 \%)$ & $120(35.71 \%)$ & $1.14(0.83-1.56)$ & 0.415 \\
\hline & Recessive & $\mathrm{A} / \mathrm{A}-\mathrm{A} / \mathrm{G}$ & $330(95.65 \%)$ & 332 (96.51\%) & 1 & & 318 (94.93\%) & 317 (94.35\%) & 1 & \\
\hline & & $\mathrm{G} / \mathrm{G}$ & 15 (4.35\%) & 12 (3.49\%) & $1.26(0.58-2.73)$ & 0.560 & 17 (5.07\%) & 19 (5.65\%) & $0.88(0.45-1.74)$ & 0.722 \\
\hline & Log-additive & - & - & - & $0.95(0.73-1.24)$ & 0.713 & - & - & $1.07(0.83-1.38)$ & 0.596 \\
\hline
\end{tabular}

Cl confidence interval, OR odds ratio, SNP single nucleotide polymorphism

$p$ values were calculated by unconditional logistic regression adjusted by age; $p<0.05$ indicates statistical significance

Highlighted in bold indicates the significant association between SNPs and breast cancer risk

Table 5 Correlations between CASC16 polymorphisms and clinical characteristics of patients with breast cancer (adjusted by age)

\begin{tabular}{|c|c|c|c|c|c|c|c|c|c|c|}
\hline \multirow[t]{2}{*}{ SNP } & \multirow[t]{2}{*}{ Genotype } & \multicolumn{3}{|c|}{ Clinical stage } & \multicolumn{3}{|c|}{ Tumor size $(\mathrm{cm})$} & \multicolumn{3}{|l|}{ LN metastasis } \\
\hline & & $\|I, I V / I\|$, & OR $(95 \% \mathrm{Cl})$ & $p$-value & $>2 / \leq 2$ & OR $(95 \% \mathrm{Cl})$ & $p$-value & Positive/Negative & OR $(95 \% \mathrm{Cl})$ & $p$-value \\
\hline \multirow[t]{6}{*}{ rs3803662 } & $A$ & $213 / 447$ & 1 & & $568 / 191$ & 1 & & $454 / 450$ & 1 & \\
\hline & G & $87 / 195$ & $0.94(0.69-1.27)$ & 0.668 & $250 / 87$ & $0.97(0.72-1.30)$ & 0.819 & $192 / 212$ & $0.90(0.71-1.14)$ & 0.368 \\
\hline & $\mathrm{AA}$ & $71 / 151$ & 1 & & $191 / 67$ & 1 & & $153 / 149$ & 1 & \\
\hline & GA & $71 / 145$ & $1.02(0.68-1.53)$ & 0.917 & $186 / 57$ & $1.13(0.0 .75-1.71)$ & 0.546 & $148 / 152$ & $0.95(0.69-1.31)$ & 0.744 \\
\hline & GG & $8 / 25$ & $0.66(0.28-1.53)$ & 0.331 & $32 / 15$ & $0.73(0.37-1.44)$ & 0.367 & $22 / 30$ & $0.71(0.39-1.30)$ & 0.270 \\
\hline & $\mathrm{GA}+\mathrm{GG}$ & $79 / 170$ & $0.97(0.66-1.43)$ & 0.869 & $218 / 72$ & $1.05(0.71-1.55)$ & 0.803 & $170 / 182$ & $0.91(0.67-1.24)$ & 0.547 \\
\hline \multirow[t]{6}{*}{ rs4784227 } & C & $206 / 479$ & 1 & & $589 / 200$ & 1 & & $447 / 491$ & 1 & \\
\hline & $\mathrm{T}$ & $94 / 163$ & $1.34(0.99-1.81)$ & 0.056 & $219 / 76$ & $0.98(0.72-1.33)$ & 0.889 & 197/161 & $1.34(1.05-1.72)$ & 0.018 \\
\hline & CC & 73/177 & 1 & & $220 / 69$ & 1 & & $155 / 185$ & 1 & \\
\hline & $\mathrm{TC}$ & $60 / 125$ & $1.19(0.79-1.81)$ & 0.400 & $149 / 62$ & $0.77(0.51-1.15)$ & 0.194 & $137 / 121$ & $1.35(0.98-1.87)$ & 0.069 \\
\hline & $\Pi$ & $17 / 19$ & $2.19(1.08-4.46)$ & 0.031 & $35 / 7$ & $1.59(0.67-3.73)$ & 0.292 & $30 / 20$ & $1.79(0.98-3.28)$ & 0.059 \\
\hline & $\mathrm{TC}+\mathrm{TT}$ & $77 / 144$ & $1.33(0.90-1.96)$ & 0.155 & $184 / 69$ & $0.85(0.58-1.25)$ & 0.410 & $167 / 141$ & $1.41(1.04-1.93)$ & 0.028 \\
\hline \multirow[t]{6}{*}{ rs45544231 } & G & $245 / 532$ & 1 & & $661 / 209$ & 1 & & $526 / 522$ & 1 & \\
\hline & C & $55 / 110$ & $1.09(0.76-1.55)$ & 0.652 & $157 / 69$ & $0.72(0.52-0.99)$ & 0.045 & $120 / 140$ & $0.85(0.65-1.12)$ & 0.244 \\
\hline & $\mathrm{GG}$ & $100 / 223$ & 1 & & $267 / 86$ & 1 & & $215 / 211$ & 1 & \\
\hline & CG & $45 / 86$ & $1.14(0.74-1.76)$ & 0.549 & $127 / 37$ & 1.09 (0.70-1.70) & 0.693 & $96 / 100$ & $0.94(0.67-1.32)$ & 0.726 \\
\hline & CC & $5 / 120$ & $0.89(0.30-2.61)$ & 0.833 & $15 / 16$ & $0.29(0.14-0.61)$ & 0.001 & $12 / 20$ & $0.59(0.28-1.23)$ & 0.160 \\
\hline & $C G+C C$ & $50 / 206$ & $1.11(0.73-1.69)$ & 0.621 & $142 / 53$ & $0.85(0.57-1.27)$ & 0.424 & $108 / 120$ & $0.88(0.64-1.22)$ & 0.450 \\
\hline \multirow[t]{6}{*}{ rs12922061 } & C & $208 / 470$ & 1 & & $583 / 203$ & 1 & & $444 / 490$ & 1 & \\
\hline & $\mathrm{T}$ & $92 / 172$ & $1.21(0.89-1.63)$ & 0.217 & $235 / 75$ & 1.09 (0.80-1.48) & 0.576 & $202 / 172$ & $1.30(1.02-1.65)$ & 0.034 \\
\hline & $\mathrm{CC}$ & $73 / 172$ & 1 & & $212 / 72$ & 1 & & $152 / 181$ & 1 & \\
\hline & $\mathrm{TC}$ & $62 / 126$ & $1.18(0.78-1.78)$ & 0.424 & $159 / 59$ & $0.93(0.62-1.34)$ & 0.709 & $140 / 128$ & $1.30(0.94-1.80)$ & 0.108 \\
\hline & $\Pi$ & $15 / 23$ & $1.58(0.78-3.20)$ & 0.209 & $38 / 8$ & $1.64(0.73-3.68)$ & 0.232 & $31 / 22$ & $1.68(0.93-3.02)$ & 0.084 \\
\hline & $\mathrm{TC}+\mathrm{TT}$ & $77 / 149$ & $1.24(0.84-1.84)$ & 0.275 & $197 / 67$ & $1.24(0.84-1.84)$ & 0.955 & $171 / 150$ & $1.34(0.99-1.85)$ & 0.051 \\
\hline \multirow[t]{6}{*}{ rs3112612 } & A & $245 / 532$ & 1 & & $661 / 207$ & 1 & & $526 / 520$ & 1 & \\
\hline & G & $55 / 110$ & $1.09(0.76-1.55)$ & 0.652 & $157 / 69$ & $0.71(0.52-0.98)$ & 0.039 & $120 / 140$ & $0.85(0.65-1.11)$ & 0.233 \\
\hline & AA & $100 / 223$ & 1 & & $267 / 85$ & 1 & & $215 / 210$ & 1 & \\
\hline & GA & $45 / 86$ & $1.14(0.74-1.76)$ & 0.549 & $127 / 37$ & $1.08(0.69-1.68)$ & 0.739 & $96 / 100$ & $0.94(0.67-1.31)$ & 0.702 \\
\hline & $\mathrm{GG}$ & $5 / 12$ & $0.89(0.30-2.61)$ & 0.833 & $15 / 16$ & $0.28(0.14-0.60)$ & 0.001 & $12 / 20$ & $0.58(0.28-1.23)$ & 0.155 \\
\hline & $G A+G G$ & $50 / 98$ & $1.11(0.73-1.69)$ & 0.621 & $142 / 53$ & $0.84(0.56-1.25)$ & 0.386 & $108 / 120$ & $0.88(0.64-1.21)$ & 0.429 \\
\hline
\end{tabular}

$p$ values were calculated by unconditional logistic regression adjusted by age; $p<0.05$ indicates statistical significance 
Table 6 The associations between CASC16 polymorphisms and BMI of breast cancer patients (adjusted by age and BMI)

\begin{tabular}{|c|c|c|c|c|c|c|c|}
\hline \multirow[t]{2}{*}{ SNP } & \multirow[t]{2}{*}{ Genotype } & \multicolumn{3}{|l|}{$>24 \mathrm{~kg} / \mathrm{m}^{2}$} & \multicolumn{3}{|l|}{$\leq 24 \mathrm{~kg} / \mathrm{m}^{2}$} \\
\hline & & Case/Control & OR $(95 \% \mathrm{Cl})$ & $p$ & Case/Control & OR $(95 \% \mathrm{Cl})$ & $p$ \\
\hline \multirow[t]{6}{*}{ rs3803662 } & A & $231 / 144$ & 1 & & $462 / 313$ & 1 & \\
\hline & G & $105 / 84$ & $0.78(0.55-1.11)$ & 0.167 & $204 / 165$ & $0.84(0.65-1.08)$ & 0.165 \\
\hline & $\mathrm{AA}$ & $76 / 47$ & 1 & & 159/101 & 1 & \\
\hline & GA & $79 / 50$ & $0.97(0.58-1.61)$ & 0.899 & $144 / 111$ & $0.83(0.59-1.19)$ & 0.313 \\
\hline & GG & $13 / 17$ & $0.46(0.20-1.04)$ & 0.063 & $30 / 27$ & $0.73(0.41-1.30)$ & 0.287 \\
\hline & $\mathrm{GA}+\mathrm{GG}$ & $92 / 67$ & $0.84(0.52-1.36)$ & 0.481 & $174 / 138$ & $0.81(0.58-1.14)$ & 0.230 \\
\hline \multirow[t]{6}{*}{ rs4784227 } & C & $231 / 174$ & 1 & & $476 / 361$ & 1 & \\
\hline & $\mathrm{T}$ & $103 / 52$ & $1.49(1.01-2.20)$ & 0.042 & 180/117 & $1.17(0.89-1.53)$ & 0.263 \\
\hline & $\mathrm{CC}$ & $77 / 65$ & 1 & & $172 / 135$ & 1 & \\
\hline & $\mathrm{TC}$ & $77 / 44$ & $1.49(0.91-2.46)$ & 0.115 & $132 / 91$ & $1.13(0.79-1.60)$ & 0.503 \\
\hline & $\pi$ & $13 / 4$ & $2.64(0.82-8.54)$ & 0.104 & $24 / 13$ & $1.45(0.71-2.95)$ & 0.308 \\
\hline & $\mathrm{TC}+\mathrm{TT}$ & $90 / 48$ & $1.59(0.98-2.58)$ & 0.059 & $156 / 104$ & $1.17(0.83-1.63)$ & 0.366 \\
\hline \multirow[t]{6}{*}{ rs45544231 } & G & 271/181 & 1 & & $534 / 383$ & 1 & \\
\hline & C & $65 / 47$ & $0.92(0.61-1.41)$ & 0.711 & 132/97 & $0.98(0.73-1.31)$ & 0.871 \\
\hline & GG & $111 / 72$ & 1 & & $217 / 154$ & 1 & \\
\hline & $C G$ & $49 / 37$ & $0.83(0.49-1.40)$ & 0.481 & $100 / 75$ & $0.96(0.67-1.38)$ & 0.817 \\
\hline & CC & $8 / 5$ & $0.98(0.31-3.15)$ & 0.979 & 16/11 & $1.07(0.48-2.38)$ & 0.869 \\
\hline & $C G+C C$ & $57 / 42$ & $0.85(0.51-1.40)$ & 0.516 & $116 / 86$ & $0.97(0.69-1.34)$ & 0.873 \\
\hline \multirow[t]{6}{*}{ rs12922061 } & C & 229/175 & 1 & & $478 / 353$ & 1 & \\
\hline & $\mathrm{T}$ & $107 / 53$ & $1.54(1.05-2.26)$ & 0.026 & 188/127 & $1.09(0.84-1.42)$ & 0.508 \\
\hline & CC & $78 / 62$ & 1 & & $166 / 129$ & 1 & \\
\hline & $\mathrm{TC}$ & $73 / 51$ & $1.15(0.70-1.88)$ & 0.581 & $146 / 95$ & $1.20(0.85-1.70)$ & 0.306 \\
\hline & $\pi$ & $17 / 1$ & $13.41(1.74-103.6)$ & 0.013 & $21 / 16$ & $1.01(0.51-2.02)$ & 0.968 \\
\hline & $\mathrm{TC}+\mathrm{TT}$ & $90 / 52$ & $1.39(0.86-2.25)$ & 0.178 & $167 / 111$ & $1.17(0.84-1.64)$ & 0.351 \\
\hline \multirow[t]{6}{*}{ rs3112612 } & A & $271 / 181$ & 1 & & $532 / 382$ & 1 & \\
\hline & G & $65 / 47$ & $0.92(0.61-1.41)$ & 0.711 & $132 / 98$ & $0.97(0.72-1.30)$ & 0.823 \\
\hline & $\mathrm{AA}$ & $111 / 72$ & 1 & & 216/154 & 1 & \\
\hline & GA & $49 / 37$ & $0.83(0.49-1.40)$ & 0.481 & $100 / 74$ & $0.97(0.68-1.40)$ & 0.887 \\
\hline & GG & $8 / 5$ & $0.98(0.31-3.15)$ & 0.979 & $16 / 12$ & $0.99(0.47-2.18)$ & 0.994 \\
\hline & $G A+G G$ & $57 / 42$ & $0.85(0.51-1.40)$ & 0.516 & $116 / 86$ & $0.98(0.69-1.38)$ & 0.896 \\
\hline
\end{tabular}

$p$ values were calculated by unconditional logistic regression adjusted by age and $\mathrm{BMl} ; p<0.05$ indicates statistical significance

Highlighted in bold indicates the significant association between SNPs and breast cancer risk

stage, tumor size, and BMI. Taken together, these findings suggested an important role for the CASC16 gene in the occurrence of breast cancer.

Rs3803662 was identified SNP in the CASC16 gene association with breast cancer as previously published studies (Udler et al. 2010). Considerably increased association between rs3803662 in the CASC16 gene and breast cancer was studied in Japanese and Caucasian women (Low et al. 2013) (Guan et al. 2016). In contrast, our present study indicated that rs3803662 played a protective role in $\mathrm{BC}$ risk $(\mathrm{OR}=0.69, p=0.042)$ in a Northwest Chinese population, and the same finding was showed in patients $\leq 50$ years $(\mathrm{OR}=0.53, p=0.014)$.
However, Edward A et al. suggested that no relationship was found between rs3803662 and breast cancer in African-American population (Ruiz-Narvaez et al. 2010). The SNP rs12922061, located in the first intron of LOC643714, was identified as a susceptibility variant of breast cancer in a Japanese GWAS (Huang et al. 2019). In our study, rs12922061 polymorphism was associated with an increased susceptibility to BC or patients with lymph node metastasis, age $\leq 50$ years and BMI $>24 \mathrm{~kg}$ / $\mathrm{m}^{2}$ individuals. Data from Chen's research showed that the increased association only observed in BC patients, no significant association was found in stratified subgroups in Southeast China population (Chen et al. 


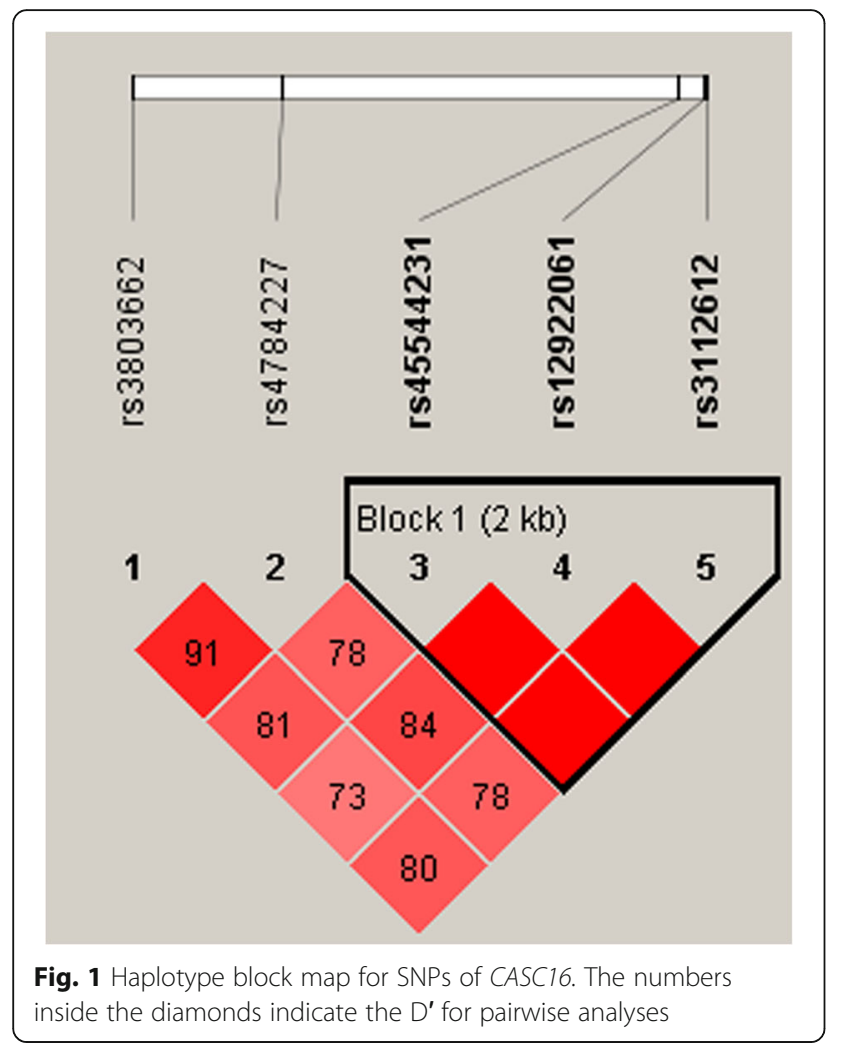

2016b). In summary, these results may be due to the differences in geography, ethnicity, and region among population, which leads to genetic variants. Our study also indicated that rs3803662 and rs12922061 played crucial roles in the progression of breast cancer.

Rs447842227 polymorphism in CASC16 is also a strong current candidate association with breast cancer risk. This study found that rs4784227 significantly increased susceptibility to breast cancer patients with age > 50 years, clinical stage III/IV, lymph node metastasis status, and BMI $>24 \mathrm{~kg} / \mathrm{m}^{2}$. These findings were in line with that of $\mathrm{He}$ (2014) who confirmed that rs4784227 could increase risk of breast cancer in a Southern Chinese population, while they hadn't identified correlation under stratified analysis (He et al. 2014) due to the difference in population. In a word, our present findings revealed that rs44842227 might be associated with age, clinical stage, lymph node metastasis status, and BMI in breast cancer.

Furthermore, our study firstly revealed that rs 45544231 and rs3112612 in CASC16 played protective roles in tumor size $>2 \mathrm{~cm}$ individuals. In addition, we also studied linkage disequilibrium (LD) and haplotype analyses of CASC16 polymorphisms in cases and controls. Haplotype analyses disclosed that $G_{\mathrm{rs} 45544231} \mathrm{~T}_{\mathrm{rs} 12922061} \mathrm{~A}_{\mathrm{rs} 3112612}$ and $\mathrm{G}_{\mathrm{rs} 45544231} \mathrm{C}_{\mathrm{rs} 12922061} \mathrm{~A}_{\mathrm{rs} 3112612}$ haplotypes reduced $\mathrm{BC}$ risk.

The major limitation of this study was the fact that we just studied the association between SCAC16 variants and breast cancer in a Northwest Chinese population. Further research in other areas or races in China is an essential step in supplementing the extant data. Besides, we determined the role of CASC16 SNPs in risk of breast cancer but there were still not detecting function of CASC16 in occurrence and evolution of breast cancer. Therefore, next work should focus on exploring the functions of CASC16 in breast cancer. In spite of its limitations, the study certainly adds to our understanding of the association between SNP variants and breast cancer. Moreover, our present work provided the possibility of using these SNPs to diagnose breast cancer in the future.

\section{Conclusions}

In summary, CASC16 rs4784227 and rs12922061 were significantly related to increased susceptibility to breast cancer. Stratification analysis revealed that rs4784227 and rs12922061 would increase BC susceptibility in age $>50$ years. Rs3803662 was a reduced factor of BC in age $\leq 50$ years. Rs4784227 was significantly improved susceptibility to BC patients in stage III/IV. The rs45544231 and rs3112612 had protective effects on BC with tumor size > $2 \mathrm{~cm}$. Rs4784227 and rs12922061 could increase BC risk in lymph node metastasis positive individuals. CASC16 rs12922061 and rs4784227 polymorphisms were correlated with increased $\mathrm{BC}$ risk in $\mathrm{BMI}>24 \mathrm{~kg} / \mathrm{m}^{2}$. We noted that $\mathrm{G}_{\mathrm{rs} 45544231} \mathrm{~T}_{\mathrm{rs} 12922061} \mathrm{~A}_{\mathrm{rs} 3112612}$ and $\mathrm{G}_{\mathrm{rs} 45544231}$ $\mathrm{C}_{\mathrm{rs12922061}} \mathrm{A}_{\mathrm{rs} 3112612}$ haplotypes reduced $\mathrm{BC}$ risk. These findings would give some new insights in the molecular mechanism of breast cancer occurrence.

Table 7 The haplotype frequencies of CASC16 polymorphisms and their associations with breast cancer risk

\begin{tabular}{|c|c|c|c|c|c|c|c|}
\hline \multirow[t]{2}{*}{$\overline{S N P}$} & \multirow[t]{2}{*}{ Haplotype } & \multicolumn{2}{|c|}{ Frequency } & \multicolumn{2}{|l|}{ Without adjusted } & \multicolumn{2}{|l|}{ With adjusted } \\
\hline & & Case & $\overline{\text { Control }}$ & $\overline{\mathrm{OR}}(95 \% \mathrm{Cl})$ & $p$ & $\overline{\mathrm{OR}(95 \% \mathrm{Cl})}$ & $p$ \\
\hline rs45544231|rs12922061|rs3112612 & CCG & 0.80 & 0.81 & $0.98(0.81-1.18)$ & 0.827 & $0.98(0.81-1.18)$ & 0.831 \\
\hline rs45544231|rs12922061|rs3112612 & GTA & 0.72 & 0.75 & $0.82(0.69-0.98)$ & 0.025 & $0.82(0.69-0.98)$ & 0.025 \\
\hline rs45544231|rs12922061|rs3112612 & GCA & 0.52 & 0.56 & $0.85(0.73-0.99)$ & 0.039 & $0.85(0.73-0.99)$ & 0.039 \\
\hline
\end{tabular}

$p$ value calculated by Wald test with and without adjusted by age

Highlighted in bold indicates the significant association between SNPs and breast cancer risk 


\section{Acknowledgements}

The authors thank all those participants and volunteers in this study. We also acknowledge the Shaanxi Provincial Cancer Hospital for their helping with sample collections.

\section{Funding}

This study was supported by the National Natural Science Foundation of China (No. 8170100875).

\section{Ethics approval and consent to participate}

All procedures performed in studies involving human participants were in accordance with the ethical standards of the Shaanxi Provincial Cancer Hospital and the 1964 Helsinki declaration.

\section{Consent for publication}

Informed consent was obtained from all individual participants included in the study.

\section{Competing interests}

All authors declare that they have no competing interests.

Received: 20 November 2019 Accepted: 15 January 2020 Published online: 29 January 2020

\section{References}

Anderson $\mathrm{GL}$, et al. Effects of conjugated equine estrogen in postmenopausal women with hysterectomy: the Women's Health Initiative randomized controlled trial. JAMA. 2004;291:1701-12.

Bray F, Ren JS, Masuyer E, Ferlay J. Global estimates of cancer prevalence for 27 sites in the adult population in 2008. Int J Cancer. 2013;132:1133-45.

Chen W, et al. Cancer statistics in China, 2015. CA Cancer J Clin. 2016a;66:115-32.

Chen $Y$, et al. The precision relationships between eight GWAS-identified genetic variants and breast cancer in a Chinese population. Oncotarget. 2016b;7: 75457-67.

FB, et al. Global cancer statistics 2018: GLOBOCAN estimates of incidence and mortality worldwide for 36 cancers in 185 countries. CA Cancer J Clin. 2018; 68:394-424.

Guan $\mathrm{H}$, et al. Detection of virus in CSF from the cases with meningoencephalitis by next-generation sequencing. J Neurovirol. 2016;22:240-5.

Han MR, et al. Genome-wide association study in east Asians identifies two novel breast cancer susceptibility loci. Hum Mol Genet. 2016a;25:3361-71.

Han W, et al. Common genetic variants associated with breast cancer in Korean women and differential susceptibility according to intrinsic subtype. Cancer Epidemiol Biomarkers Prev. 2011;20:793-8.

Han YJ, Zhang J, Zheng Y, Huo D, Olopade OI. Genetic and epigenetic regulation of TOX3 expression in breast Cancer. PLoS One. 2016b;11:e0165559.

He X, Yao G, Li F, Li M, Yang X. Risk-association of five SNPs in TOX3/LOC643714 with breast cancer in southern China. Int J Mol Sci. 2014:15:2130-41.

Huang YB, Song FJ, Chen KX. Application values of genome-wide association studies in screening for breast cancer. Zhonghua liu xing bing xue za zhi. 2019;40:713-8

Islam T, et al. Alcohol and dietary folate intake and the risk of breast cancer: a case-control study in Japan. Eur J Cancer Prev. 2013;22:358-66.

Liao J, Chen Y, Zhu J, Wang Q, Mo Z. Polymorphisms in the TOX3/LOC643714 and risk of breast cancer in South China. Int J Biol Markers. 2018;33(4): 1724600818755633

Lichtenstein P, et al. Environmental and heritable factors in the causation of cancer--analyses of cohorts of twins from Sweden, Denmark, and Finland. N Engl J Med. 2000:343:78-85.

Lilyquist J, Ruddy KJ, Vachon CM, Couch FJ. Common genetic variation and breast Cancer risk-past, present, and future. Cancer Epidemiol Biomarkers Prev. 2018;27:380-94

Low SK, et al. Genome-wide association study of breast cancer in the Japanese population. PLoS One. 2013;8:e76463

Nelson HD, et al. Risk factors for breast cancer for women aged 40 to 49 years: a systematic review and meta-analysis. Ann Intern Med. 2012;156:635-48.

Rudolph A. Gene-environment interaction and risk of breast cancer. Br J Cancer. 2016;114:125-33.

Ruiz-Narvaez EA, et al. Polymorphisms in the TOX3/LOC643714 locus and risk of breast cancer in African-American women. Cancer Epidemiol. 2010;19: $1320-7$.
Ruiz-Narvaez EA, et al. Fine-mapping of the $6 \mathrm{q} 25$ locus identifies a novel SNP associated with breast cancer risk in African-American women. Carcinogenesis. 2013;34:287-91.

Sehrawat B, et al. Potential novel candidate polymorphisms identified in genome-wide association study for breast cancer susceptibility. Hum Genet. 2011;130:529-37.

Torre LA, Islami F, Siegel RL, Ward EM, Jemal A. Global Cancer in women: burden and trends. Cancer Epidemiol Biomarkers Prev. 2017;26:444-57.

Udler MS, et al. Fine scale mapping of the breast cancer $16 q 12$ locus. Hum Mol Genet. 2010;19:2507-15.

Walsh T, King MC. Ten genes for inherited breast cancer. Cancer Cell. 2007;11:103-5.

Wang $Q$, et al. Increased risk of breast cancer in individuals carrying the TNRC9 rs3803662 C>T polymorphism: a meta-analysis of case-control studies. Genetics Mol Res. 2016;15:1-11.

Wanging $C$, et al. Cancer incidence and mortality in China. Chin J Cancer Res. 2014;30:1-12.

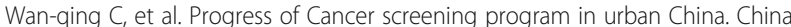
Cancer. 2019;28:15-22.

Xia P, et al. Polymorphisms in ESR1 and FL43663 are associated with breast cancer risk in the Han population. Tumour Biol. 2014;35:2187-90.

Yang P, et al. CYP17 polymorphisms are associated with decreased risk of breast cancer in Chinese Han women: a case-control study. Cancer Manag Res. 2018;10:1791-8.

Zarco MF, Vess TJ, Ginsburg GS. The oral microbiome in health and disease and the potential impact on personalized dental medicine. Oral Dis. 2012;18:109-20.

Zhou $\mathrm{L}$, et al. Association of five single nucleotide polymorphisms at 6q25.1 with breast cancer risk in northwestern China. Am J Cancer Res. 2015;5:2467-75.

\section{Publisher's Note}

Springer Nature remains neutral with regard to jurisdictional claims in published maps and institutional affiliations.

Ready to submit your research? Choose BMC and benefit from:

- fast, convenient online submission

- thorough peer review by experienced researchers in your field

- rapid publication on acceptance

- support for research data, including large and complex data types

- gold Open Access which fosters wider collaboration and increased citations

- maximum visibility for your research: over $100 \mathrm{M}$ website views per year

At $\mathrm{BMC}$, research is always in progress.

Learn more biomedcentral.com/submissions 